\title{
PISA-Studie 2018: Leseförderung muss höchste Priorität bekommen. Bibliotheken beteiligen sich am Nationalen Lesepakt
}

https://doi.org/10.1515/bd-2020-0029

Als Grundvoraussetzung aller Bildungsprozesse muss die Förderung von Lesekompetenz endlich eine Priorität der Bildungspolitik werden. Die Ergebnisse der aktuell veröffentlichten PISA-Studie sind deutlich: 20,7 Prozent der 15-Jährigen in Deutschland haben Schwierigkeiten beim Lesen. Damit führt die Studie den Befund der IGLU-Studie 2016 fort, nach der knapp ein Fünftel der Zehnjährigen in Deutschland die Inhalte, die sie lesen, nicht verstehen.

„Die Ergebnisse der PISA-Studien belegen: Lesen muss früh, systematisch und unabhängig von Einkommen, Bildungsgrad und Herkunft der Eltern gefördert werden. Dies kann nur gelingen, wenn alle Bildungseinrichtungen koordiniert zusammenarbeiten und idealerweise auch die Eltern und das familiäre Umfeld adressieren“, so Prof. Dr. Andreas Degkwitz, Bundesvorsitzender des Deutschen Bibliotheksverbands e.V. „Bibliotheken sind neben den formalen Bildungseinrichtungen die wichtigsten Akteure der Leseförderung, die alle Kinder und ihre Familien dauerhaft mit passgenauen Angeboten begleiten können. Daher beteiligt sich der Deutsche Bibliotheksverband an dem von der Stiftung Lesen initiierten Nationalen Lesepakt.“

Soll die Leseförderung erfolgreich sein, müssen die Unterstützungsangebote auf die Bedarfe der Risikogruppen abgestimmt sein und diese auch erreichen. Die PISA-Studie belegt, dass insbesondere schwächere Schüler^innen, die nicht aufs Gymnasium gehen, sowie Kinder aus Familien mit Migrationshintergrund, schlechter lesen können.

Als außerschulische Bildungsorte können Bibliotheken gerade für diese Gruppen mit systematisch aufeinander aufbauenden Angeboten wichtige Unterstützer sein - als fester Partner von Kitas, Grund- und weiterführenden Schulen. Aufgrund der Bedeutung der frühkindlichen Leseförderung (,early literacy“) für den späteren Erwerb von Lesefähigkeit verstärken Bibliotheken hier seit Jahren ihre Angebote für die unter Sechsjährigen. Neben der Bereitstellung eines breiten Medienangebots bieten Bibliotheken Kindern und Jugendlichen zahlreiche Möglichkeiten für ihre Freizeitgestaltung an und fördern mit vielfältigen Veranstaltungen digitale und analoge Lesekompetenzen sowie die Leselust.

Das Potential der Partnerschaft zwischen Bibliotheken und den formalen Bildungsinstitutionen muss erkannt und systematisch gefördert werden. Für den 
Ausbau und die Intensivierung dieser Partnerschaft bedarf es bildungspolitischer Unterstützung über die Verankerung der Kooperation in den jeweiligen Bildungsplänen der Bundesländer.

Mehr Information zur PISA-Studie 2018 finden Sie unter: https://www.oecd. org/berlin/themen/pisa-studie.

\section{Der Deutsche Bibliotheksverband e.V. (dbv)}

Der Deutsche Bibliotheksverband e.V. (dbv) vertritt mit seinen mehr als 2.100 Mitgliedern bundesweit rund 10.000 Bibliotheken mit 25.000 Beschäftigten und 11 Mio. Nutzer^innen. Sein zentrales Anliegen ist es, Bibliotheken zu stärken, damit sie allen Bürger`innen freien Zugang zu Informationen ermöglichen. Der Verband setzt sich ein für die Entwicklung innovativer Bibliotheksleistungen für Wissenschaft und Gesellschaft. Als politische Interessensvertretung unterstützt der dbv die Bibliotheken insbesondere auf den Feldern Informationskompetenz und Medienbildung, Leseförderung und bei der Ermöglichung kultureller und gesellschaftlicher Teilhabe für alle Bürgerinnen.

\section{Kontakt:}

Deutscher Bibliotheksverband e.V. (dbv)

Kristin Bäßler, Leitung Kommunikation / Pressesprecherin

Tel.: 030 / 6449899 25, E-Mail: presse@bibliotheksverband.de

URL: www.bibliotheksverband.de 